\title{
Analysis of Combustion Closure Assumptions in a Dual-Mode Scramjet Combustor
}

\author{
Wai Lee Chan* \\ Department of Aerospace Engineering, University of Michigan, Ann Arbor, MI, 48109 \\ Matthias Ihme I $^{\dagger}$ \\ Department of Mechanical Engineering, Stanford University, Stanford, CA, 94305
}

\begin{abstract}
A study to evaluate the combustion closures in the context of large-eddy simulations of a dual-mode scramjet combustor is being conducted. The geometry of the scramjet corresponds to the operating point " $A$ " of the University of Virginia's dual-mode scramjet experiment. Results from this work will contribute to ongoing efforts in advancing the incorporation of numerical predictive tools, in particular the large-eddy simulation methodologies, in the design of practical high-speed air-breathing propulsion systems. More importantly, this work will serve to provide insights to the characters of supersonic turbulent reacting flow regimes that are relevant to numerical simulations. To this end, simulations that employ two turbulence combustion models, namely the flamelet/progress variable model and the laminar finite-rate chemistry approximation, have been completed and compared against experimental data. Several combustion closure analyses are also conducted to study the effect of turbulence-chemistry interaction and the applicability of flamelet theories.
\end{abstract}

\section{Nomenclature}

\begin{tabular}{ll}
$x, y, z$ & Streamwise, wall-normal, spanwise position, $\mathrm{m}$ \\
$p$ & Pressure, Pa \\
$T$ & Temperature, $\mathrm{K}$ \\
$M_{E}$ & Pope's criterion \\
$k_{t}^{\prime}$ & Subgrid-scale kinetic energy, J/kg \\
$K_{E}$ & Resolved turbulent kinetic energy, J/kg \\
$u$ & Velocity, m/s \\
$Z$ & Mixture fraction \\
$\chi Z$ & Scalar-dissipation rate, $1 / \mathrm{s}$ \\
$C$ & Progress variable \\
$Y$ & Species mass fraction \\
$\dot{\omega}$ & Reaction rate, $1 / \mathrm{s}$ \\
Operator & \\
$\langle\cdot\rangle$ & Ensemble averaging \\
$\sim$ & Spatial filtering \\
Subscript & \\
st & Stoichiometric condition \\
$i$ & Directional indices \\
Superscript & \\
\hline,$/ / 2$ & Fluctuating quantities
\end{tabular}

${ }^{*}$ Graduate Student Research Assistant, Member AIAA.

${ }^{\dagger}$ Assistant Professor, Member AIAA. 


\section{Introduction}

In relation to long-range strike and access to orbit, air-breathing propulsion systems such as scramjet are often considered as attractive options to conventional rocket engines due to their favorable characteristics, for instance reduced payload-cost and higher specific impulse, among others. However, a widespread utilization of these systems has yet to be materialized, mainly due to the challenges involved in the design of these systems, which must account for stringent requirements to function over a wide range of operating conditions. In addition, flight-testings of supersonic air-breathing vehicles are often accompanied with prohibitive costs, whereas ground-testings are typically unable to fully reproduce realistic flight conditions. For these reasons, an attractive alternative is to make use of simulation techniques to predict the performance of these high-speed air-breathing propulsion systems. In particular, the large-eddy simulation (LES) methods have demonstrated promising capabilities to describe key features in various supersonic configurations, including dual-mode combustors ${ }^{1,2}$ and scramjet facilities. ${ }^{3,4}$ Interested readers can refer to a recent review by Fureby, ${ }^{5}$ which is dedicated to the subject of LES of supersonic combustion.

However, since the consideration of complex flow dynamics, such as shocks, turbulence-chemistry interactions (TCI), and flame instabilities, is equally relevant to numerical simulations, the incorporation of computational techniques in scramjet design requires validation studies. The University of Virginia's dualmode scramjet experiments are designed exactly to accomplish this requirement. Supported by the National Center for Hypersonic Combined-Cycle Propulsion program, ${ }^{6,7}$ these scramjet experiments, performed at the University of Virginia's Supersonic Combustion Facility, have contributed to a unique and extensive set of benchmark data that will greatly benefit numerical model validations. Non-intrusive diagnostic techniques that have been implemented include focused Schlieren and stereoscopic particle image velocimetry (SPIV), ${ }^{7}$ coherent anti-Stokes Raman spectroscopy (CARS), ${ }^{8}$ and planar laser induced fluorescence (PLIF), ${ }^{9}$ providing measurements of density gradient, velocity fields, hydroxyl radical concentration, temperature, and species mole fractions.

The objective of the current study is to perform a numerical investigation on the operating point "A" of the University of Virginia's (UV "A") dual-mode scramjet experiment. So far, there have been several other numerical studies on this particular configuration, ${ }^{2,3,10,11}$ exploring different topics such as the sensitivity of the simulation towards reaction mechanisms, the influence of TCI, and the applicability of various turbulence closures. However, it is clear that these studies are mainly result-oriented, meaning that their true interests lie in generating solutions that will replicate the experimental measurements. Therefore, it is desirable that further numerical studies on the UV "A" scramjet combustor be steered towards understanding the intrinsic characters of the configuration.

In order to meet the objective, this work will consider a comparative study of two turbulence combustion models, namely the flamelet/progress variable (FPV) combustion model ${ }^{12,13}$ and laminar finite-rate chemistry (FR) approximation. All simulations are performed with a compressible LES solver, CHRIS, ${ }^{14}$ and a detailed hydrogen-air reaction mechanism. ${ }^{15}$ Available and crucial augmentations to the solver will be incorporated to ensure optimal calculations. Additionally, the computations will be performed using identical grid-quality, subgrid turbulence closure, and time-advancing scheme. Hence, any changes in the simulation results will be due to the intended parametric modification, thus guaranteeing a consistent assessment.

The geometry, boundary and operating conditions, and computational setup that are relevant to the UV "A"-configuration are presented in the next section. Statistical results from the experiment and both simulations are compared in Sec. III, followed by an analysis of the effects of the combustion model by cross-referencing the two numerical datasets. Then, the paper will discuss the findings and their significance, and finishes with conclusions.

\section{Experimental Configuration and Computational Setup}

The geometry and boundary conditions of the computational domain are shown in Figs. 1(a)-1(b). Note, from Fig. 1(a), that this domain covers only part of the entire UV "A" scramjet configuration, namely the isolator, the combustor, and the extender sections. Instead of including the upstream convergingdiverging nozzle, a uniform flow of air with streamwise velocity of $1035 \mathrm{~m} / \mathrm{s}$ (corresponding to a Mach-2, flow based on the static thermodynamic state of $p=38 \mathrm{kPa}$ and $T=667 \mathrm{~K}$ ) is imposed at the inflow plane. The fuel injection is described by a mean uniform flow of pure hydrogen with injection speed of $1783 \mathrm{~m} / \mathrm{s}$ through a port with diameter $d=2.54 \mathrm{~mm}$, corresponding to a Mach-1.7 condition, based on static 
pressure and temperature of $94 \mathrm{kPa}$ and $190 \mathrm{~K}$, respectively. Artificially generated turbulence is introduced to the mean fuel-flow to represent the turbulent flow dynamics of the fuel jet. The global equivalenceratio that corresponds to these inflow conditions is 0.17 , indicating a fuel-lean combustion regime. All walls are prescribed by the no-slip and adiabatic conditions. The outflow plane is assigned by convectiveoutflow conditions. Figure 1(c) shows the isometric view of the UV "A" scramjet combustor, emphasizing the principal planes-of-interest, namely the centerplane $(z=0)$ and two cross-sections $(x=7.5 \mathrm{~cm}$ and $x=11.3 \mathrm{~cm}$ ), which coincide with two of the four CARS-measurement locations. ${ }^{8}$

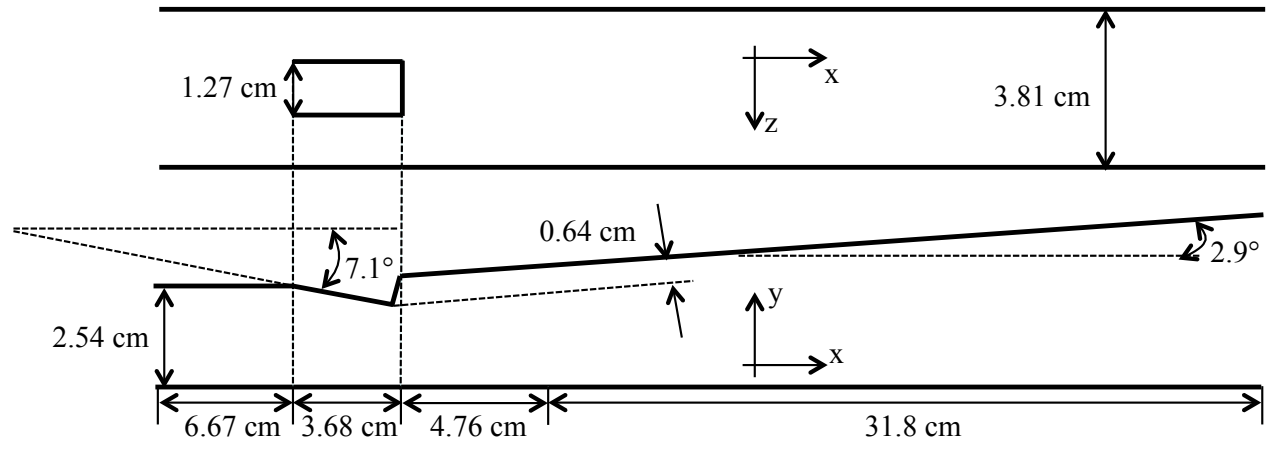

(a)

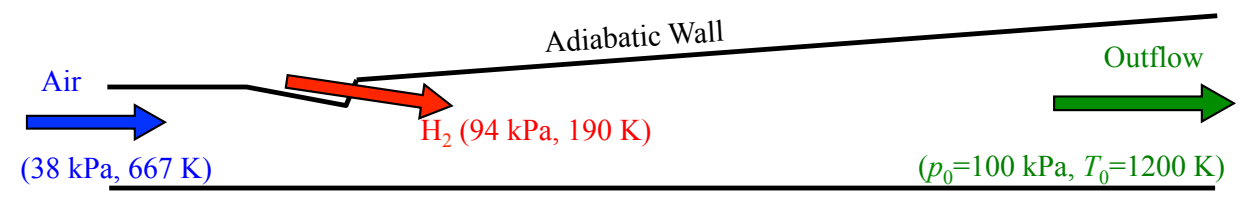

Adiabatic Wall

(b)

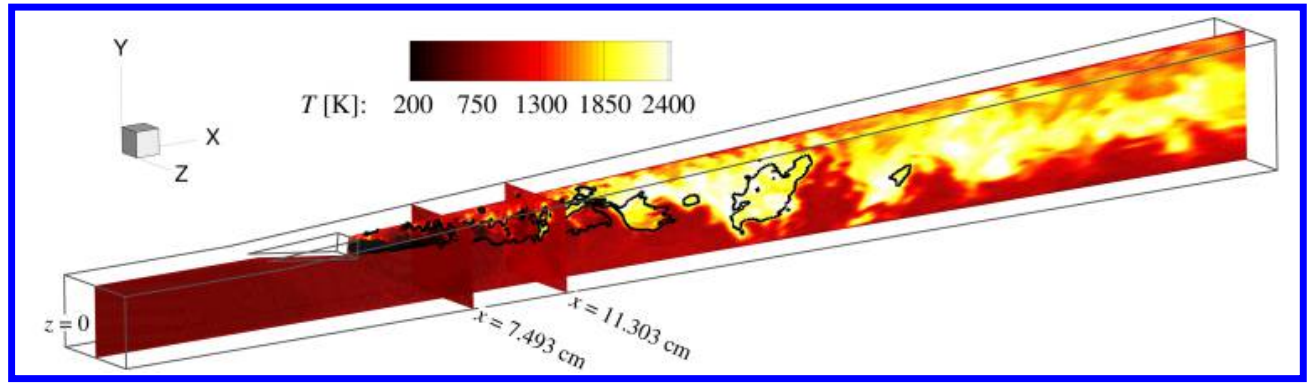

(c)

Figure 1. Schematic illustration of the geometry (top) and boundary conditions (middle) of the UV "A" scramjet configuration. As indicated by the various arrows, the general direction of the bulk flow is from left to right. An isometric view of the configuration is provided in the bottom figure, showing the principal planes-of-interest. The fuel injection port (not shown) has a diameter of $d=2.54 \mathrm{~mm}$.

The computational domain is discretized by a mixed hexagonal-prism mesh that consists of approximately 40 million control volumes. The ratio of prism to hexagonal elements is kept minimal so that the grid is largely regular. The flow-through-time is defined with respect to the length of the computational domain and the fuel injection speed, and is equal to $0.21 \mathrm{~ms}$.

Currently, the turbulence closures of the filtered momentum and filtered mixture fraction variance equations are provided by the Vreman eddy-viscosity subgrid-scale (SGS) model ${ }^{16}$ and spectral arguments, respectively. In addition, TCI is either: (i) closed by the FPV combustion model ${ }^{12,13}$ or (ii) omitted by the FR-closure. The reaction chemistry is represented by a detailed hydrogen-air mechanism consisting of nine species and 19 elementary reactions. ${ }^{15}$ The state-space trajectory that corresponds to the setup is presented in the form of a "S"-shaped curve in Fig. 2, which indicates the crossover temperature ${ }^{17}$ and quenching scalar-dissipation rate are $910 \mathrm{~K}$ and $250 \mathrm{1} / \mathrm{s}$, respectively. For further details, like the governing equations of the LES and the characteristics of the combustion model, the reader is referred to our previous work. ${ }^{11}$ 


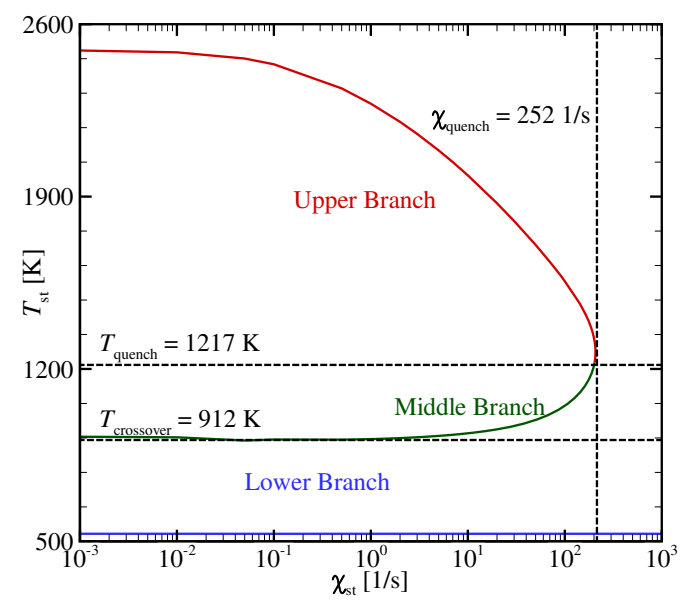

Figure 2. "S"-shaped curve derived from the prescribed boundary and operating conditions and reaction mechanism. ${ }^{15}$

\section{Results}

Prior to the demonstration of the simulation results, it is useful to first evaluate the level of grid-resolution that has been utilized in the LES. To do so, we refer to Pope's criterion: ${ }^{18}$

$$
M_{E}=\frac{\left\langle\widetilde{k}_{t}\right\rangle}{\left\langle\widetilde{K}_{E}\right\rangle+\left\langle\widetilde{k}_{t}\right\rangle}
$$

where $\widetilde{k}_{t}$ is the subgrid-scale kinetic energy, provided by the SGS model, $\widetilde{K}_{E}=\left(\widetilde{u}_{i} \widetilde{u}_{i}-\left\langle\widetilde{u}_{i}\right\rangle\left\langle\widetilde{u}_{i}\right\rangle\right) / 2$ is the turbulent kinetic energy of the resolved scales. The value of $M_{E}$ is bounded between 0 and 1 , corresponding to a direct numerical (all scales resolved) and a Reynolds-averaged Navier-Stokes (all scales modeled) simulation, respectively. Pope suggested that $M_{E} \leq 0.2$ (a resolution of $80 \%$ of the total kinetic energy) is an appropriate standard for LES. Pope's criterion of the simulations, computed with the non-reacting solution, is illustrated in Fig. 3, showing that the threshold of $M_{E} \leq 0.2$ is satisfied for a dominant part of the computational domain. The areas where $M_{E}>0.2$ coincide with the vicinities of shocks and expansion waves. Note that only the non-reacting $M_{E}$-profiles is presented since combustion has been found to generally reduce turbulent scales and thus decrease $M_{E}$ values, rendering the non-reacting case limiting for Pope's criterion. Thus, the current grid is regarded sufficient for LES of the UV "A" configuration, particularly since regions critical for chemical reactions are all within Pope's $M_{E}$-threshold. One caveat in the utility of Pope's criterion is the singular behavior of $M_{E}$ in laminar-flow regimes, where the denominator in Eq. (1) will approach zero. For this reason, regions in where $\left\langle\widetilde{K}_{E}\right\rangle \leq 1 \mathrm{~J} / \mathrm{kg}$ have been blanked out so that large $M_{E}$ corresponding to laminar flow regions is differentiated from that caused by insufficient resolution.

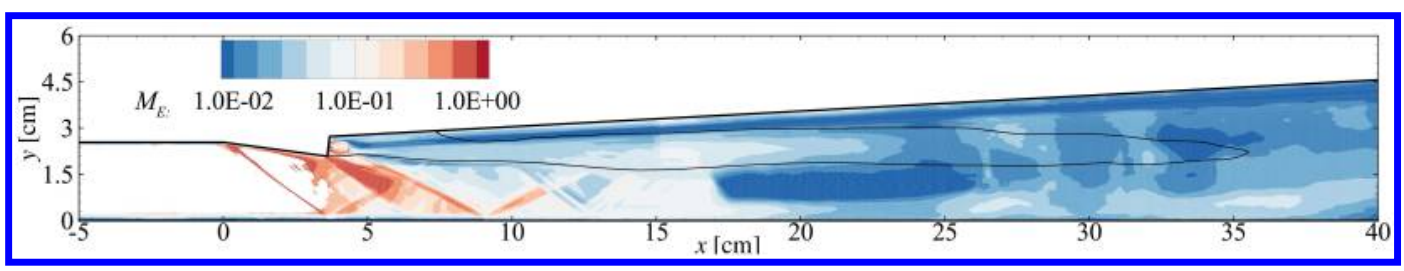

Figure 3. Pope's criterion ${ }^{18} M_{E}$ of the non-reacting case along the centerplane. The black line denotes the mean stoichiometric mixture fraction $\langle\widetilde{Z}\rangle=Z_{\text {st }}=0.0285$ iso-line.

Qualitative comparisons of the non-reacting simulations and measured ensemble-averaged temperature profiles at $x=15.2 \mathrm{~cm}$ are shown in Fig. 4 . Note that this will be the only point where we refer to a plane other than the aforementioned three principal planes, for the reason that non-reacting measurements are only available at this stated location. Noticeably, the two results are not in a good agreement, with the simulation results showing much more homogeneity and symmetry than their experimental counterparts. These discrepancies may be attributed to the thermal non-equilibrium and asymmetric flow conditions ${ }^{8}$ 
upstream of the combustor section, which were neglected in the simulations. In addition, the simulated jet plume, indicated by the inner area of high temperature standard deviations, is more compact and exhibits a well-defined counter-rotating vortical structure, as opposed to that of the experiment. These observations indicate that the LES solver has a tendency to underpredict the mixing rate of the flow. While a more realistic inflow boundary condition that accounts for the non-uniform heating in the facility ${ }^{8}$ may alleviate such underprediction, the improvement is postulated to be minimal in the reacting case. Therefore, we retained the simple inflow boundary condition, noting the potential underprediction of the mixing rate that is associated with such simplifications.

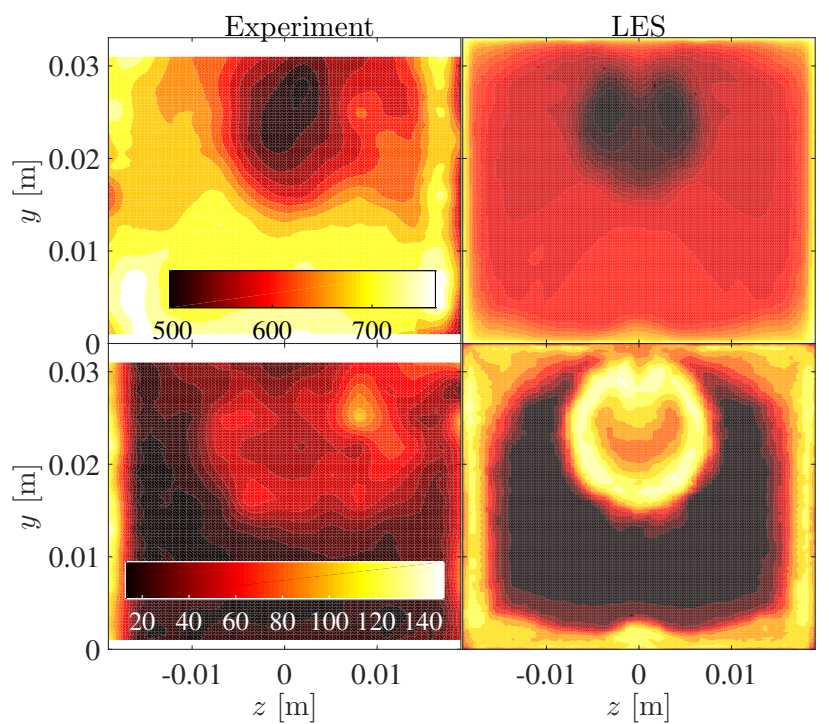

Figure 4. Cross-sectional profiles of mean temperature (top) and RMS (bottom) at $x=15.2 \mathrm{~cm}$ for the non-reacting case.

In contrast to the non-reacting results, the results from the reacting flow simulation are in substantially better qualitative agreement with the CARS-measurements, as shown in Figs. 5-6. From Fig. 5, the FPVresult is in slightly better agreement than its FR counterpart in preserving a toroidal flame structure that is hotter on the upper side, but clearly underpredicts the reactant-mixing rate, resulting in a larger fuel core than the CARS-profile. This undermixed core is indicated by the area of low temperature within the toroid. In contrast, the FR-model noticeably overpredicts the reaction-rate, exhibiting a hotter fuel core and thus less-defined flame-ring than the other two results. Both simulations have apparently higher peak RMS values than the experiment, suggesting that the numerical temperature fluctuations are more dominant than the physical fluctuations. The spreading of the RMS profiles indicates that the spatial-range of intermittency of the FR-case is closer to the experimental measurements than that of the FPV-closure.

A general improvement in the discrepancies with CARS-measurements is observed with increasing streamwise distance, as indicated by comparing the results at $x=11.3 \mathrm{~cm}$, shown in Fig. 6 , to that at $x=7.5 \mathrm{~cm}$ of Fig. 5. For instance, the spanwise spreading of the flame ranges approximately between $z= \pm 1.5 \mathrm{~cm}$ for both experimental and simulation results. Also, the peak fluctuation intensity, which resides within the mixing layer, is approximately $20 \%$ of the maximum mean temperature value for all results. While the aforementioned underprediction of the mixing rate in the FPV-result is still discernible in Fig. 6, the differences from the CARS-measurement is significantly smaller than seen in Fig. 5. On the contrary, the overprediction of the reaction-rate by the FR-model at $x=7.5 \mathrm{~cm}$ seemingly has no significant effect on the current downstream plane, where the FR mean temperature exhibits the smallest flame area and lowest value among the three results. The last observation suggests that the omission of TCI in the FR-case may not be as important downstream as it is at the flameholding recirculation region $(3 \lesssim x \lesssim 7 \mathrm{~cm})$; a further discussion on this subject is given in the following section.

The top-wall pressure distribution, which is relevant to the amount of thrust generated from a scramjet, is evaluated in Fig. 7. Clearly, the simulations provide reasonably accurate predictions for this measure, showing a generally good agreement with the measurements (indicated by open and filled symbols for the mixing and reacting cases, respectively). In the region between $5<x<7 \mathrm{~cm}$, the reacting FPV-result (thin solid 


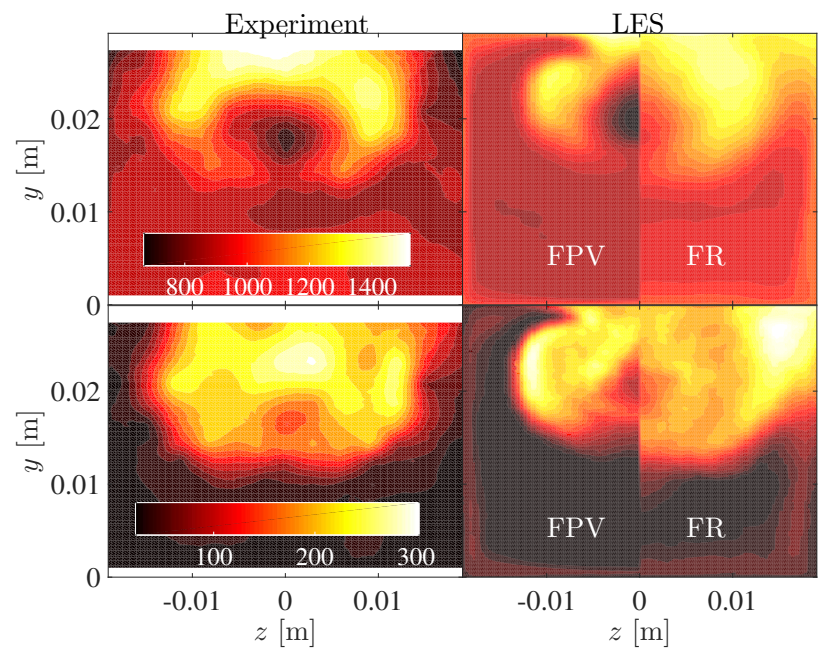

Figure 5. Cross-sectional profiles of mean temperature (top) and RMS (bottom) at $x=7.5 \mathrm{~cm}$ for the reacting case.

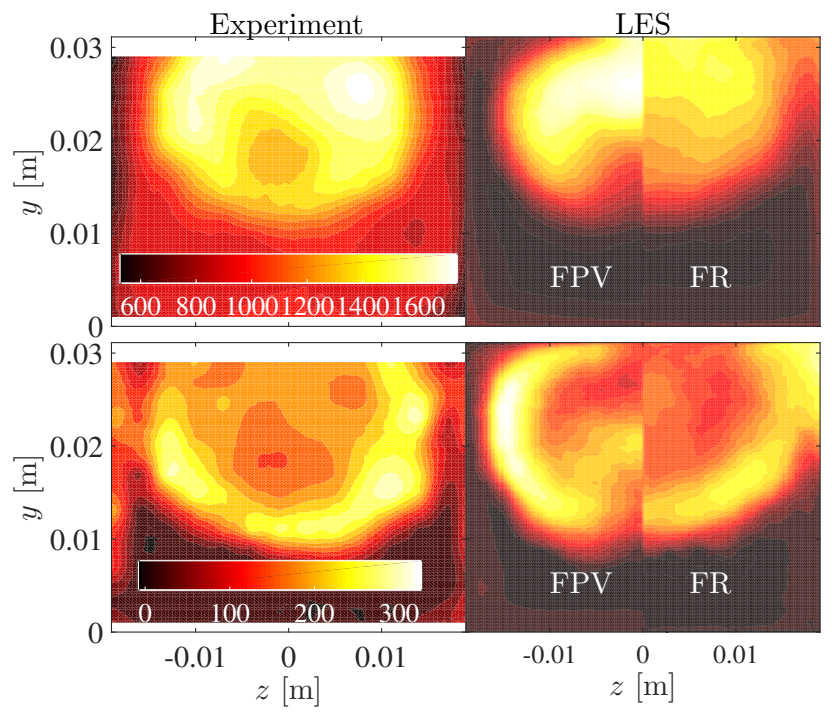

Figure 6. Cross-sectional profiles of mean temperature (top) and RMS (bottom) at $x=11.3 \mathrm{~cm}$ for the reacting case.

line) shows an underprediction of approximately 30\%. Conversely, the reacting FR-result (thickened solid line) appears to agree excellently with the measurement in the same region, but subsequently overpredicts the pressure by approximately $8 \%$ at $7<x<15 \mathrm{~cm}$. This behavior of the FR-result indicates that: (i) the underprediction by the FPV-model is attributed to the aforementioned misrepresentation in mixing and can benefit from an increase in the reaction-rate; and (ii) the reaction-rate in the FR-case is overcompensating, hence is likely to be higher than that in the experiments. Beyond $x \approx 15 \mathrm{~cm}$, both simulation results agree well with the measurements and each other, substantiating the claim of a general improvement with streamwise distance.

Interestingly, comparable over- and underpredictions in the pressure distribution are also observed in the numerical studies of Fulton et al. and Hassan et al., ${ }^{2,10}$ suggesting that these errors are relatively universal to CFD-technique on this configuration, regardless of reaction mechanism or closure model. Insight may be shed on this observation by comparing the non-reacting and reacting solutions. For instance, the non-reacting simulated result between $5<x<15 \mathrm{~cm}$, where the errors of the reacting cases are most severe, is in discernibly good agreement with the corresponding non-reacting measurements, suggesting that an inadequate/excessive amount of reaction may be the root of the under/overprediction of the top-wall 
pressure. This inaccurate description of the reaction is possibly a consequence of the misrepresentation of the mixing rate, as suggested by the aforementioned misrepresentation of mixing in the FPV-case. On the other hand, the underprediction in the non-reacting simulation result between $20<x<30 \mathrm{~cm}$ may be attributed to an inadequate grid-resolution, since a streamwise grid-stretching has been implemented. The reacting simulation, however, is less sensitive the grid-stretching because combustion tends to relax the requirement on the grid-resolution, as has been discussed in the Pope's criterion analysis.

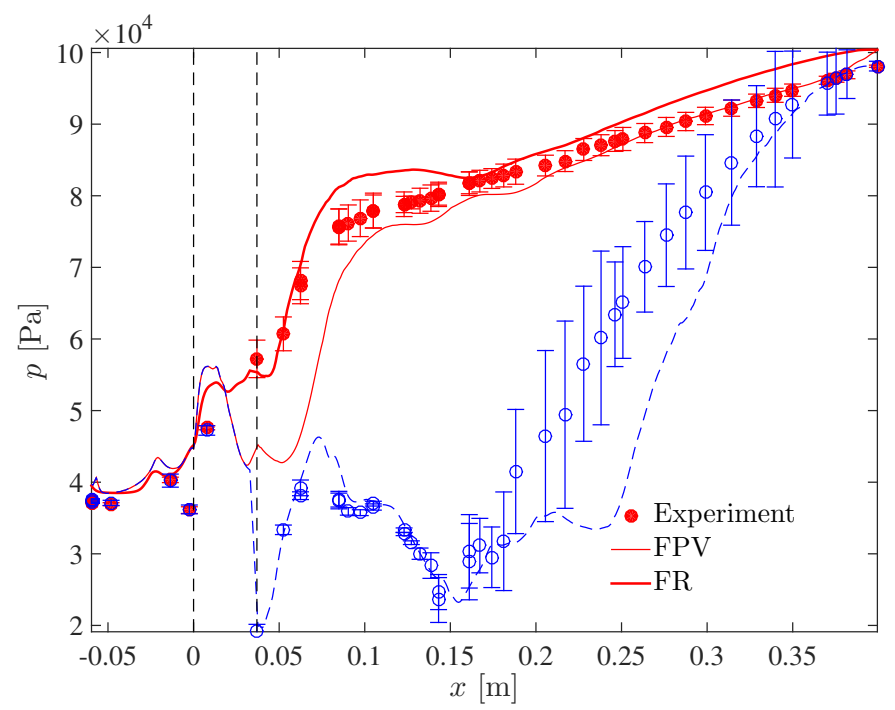

Figure 7. Top-wall pressure distribution in the streamwise-direction for the reacting (solid lines, filled symbol) and mixing (dashed line, open symbol) cases. The error bars of the measurements correspond to the 95\% confidenceintervals. The two vertical lines, in increasing $x$, denote the compression ramp leading-edge and fuel-injection port. Only one line is shown for the mixing case since the combustion model is irrelevant.

Despite their discrepancies from the measurements, both simulations are able to capture the essential physical behaviors of the configuration, and therefore can be utilized to extract insights to the characterization of the UV "A" scramjet combustor. To do so, analyses that compare the similarities and differences of the two simulation results are conducted in the next section.

\section{Turbulence Combustion Model Analyses}

In the following, the sensitivity of the simulations to the turbulence combustion model will be assessed, starting with the global characteristic of the simulations as indicated by the mean centerplane temperature distribution of the scramjet, shown in Fig. 8. Consistent with the cross-sectional analyses, the FR-simulation predicts a noticeably higher upstream reaction-rate than the FPV-case, as indicated by the shorter fueljet penetration and earlier temperature rise. The initial is characterized by the area of low temperature $(T<500 \mathrm{~K})$, while the latter is denoted by the mean streamwise location where temperature first exceeds the crossover temperature $T_{\text {crossover }}=1000 \mathrm{~K}$. Such overprediction in the reaction-rate by the FR-model is possibly related to the omission of turbulence-reaction coupling, which will suppress the reaction-rate at the fuel injection vicinity with intense mixing and high strain on the reactants.

From Fig. 8, the FR-result can be seen to increase at a lower rate (i.e. smaller $\partial T / \partial x$ ) than its FPV counterpart, despite exceeding crossover temperature earlier than the FPV temperature. This slower rise in temperature is also reflected in Fig. 6, where the temperature in the FR-result is lower than both measurements and FPV-solutions, and is attributed to the localization of the overprediction in reaction-rate by the FR-model at the flameholding recirculation region (window nearer to the fuel injection point in Fig. 8). The downstream insensitivity to these upstream discrepancies, introduced in the top-wall pressure comparison (cf. Fig. 7), is discernable in the current results as well, where the two simulations are qualitatively similar beyond $x=15 \mathrm{~cm}$. This loss of upstream conditions is consistent with the general improvement with increasing streamwise distance in both results, as discussed in Sec. III.

In order to assess the effects of TCI, the mean centerplane distribution of the reaction-rate of $\widetilde{Y}_{\mathrm{H}_{2} \mathrm{O}}$, which corresponds to the progress variable $\widetilde{C}$, is illustrated in Fig. 9; note that reaction-rates with absolute 


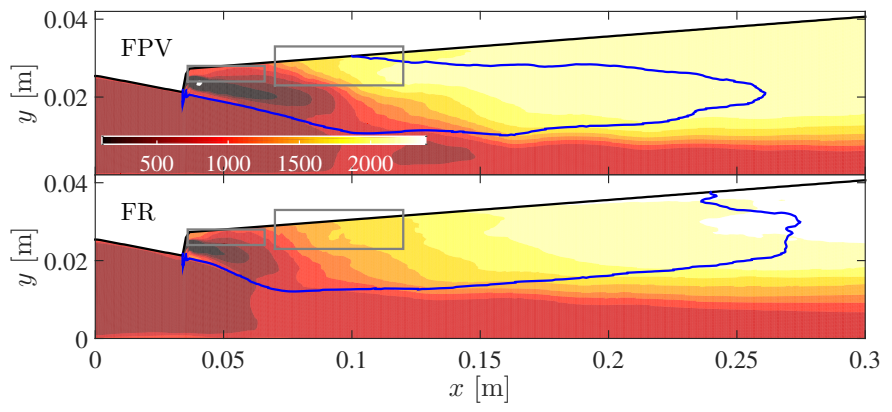

Figure 8. Mean temperature profile of the FPV- (top) and FR-cases (bottom) along the centerplane. The thickened iso-line refers to $\widetilde{Z}=Z_{\text {st }}=0.0285$, while the boxes denote the windows where probabilistic information are taken from for the analysis shown in Fig. 10, representing, in increasing $x$, the recirculation and flame-stabilization regions.

values less than 10 1/s has been blanked for clarity. While the reaction-rate of the FR-case occurs at further upstream location than that of the FPV-model, the two profiles are similar in terms of their shape, as though they are set out-of-phase by an axial-translation. Recognizing that the reaction-rate of the FPV-case is parameterized by a lower-order manifold, which consists of $\widetilde{Z}, \widetilde{Z^{\prime \prime 2}}$, and $\widetilde{C}$, while that of the FR-case is evaluated through a finite-rate chemical mechanism that involves all species, this similarity suggests that: (i) the FPV parameterization is a reasonable representation of the detailed chemistry; and (ii) the discrepancies between the two simulations are attributed to their differences in the transport of the three scalars of the FPV-manifold. Considering the consistent implementation of the two simulations, the differences in scalar transport are then likely a result of the omission of TCI by the FR-model, which can be quantified by closing in to the region where TCI is significant, which is indicated in Fig. 9 by the thickened iso-line. For convention, this region is defined by the mixedness condition: $\widetilde{Z^{\prime \prime 2}}>0.005(\widetilde{Z}(1-\widetilde{Z}))$, using the FPV-results. In accordance to a seperate correlation study (not shown), the FPV-to-FR reaction-rate ratio, conditioned on this high TCI region, is best-fitted by a linear gradient of approximately 0.22 , thereby quantifying the overprediction of the reaction-rate by the FR-closure as approximately four times that of the FPV-model.

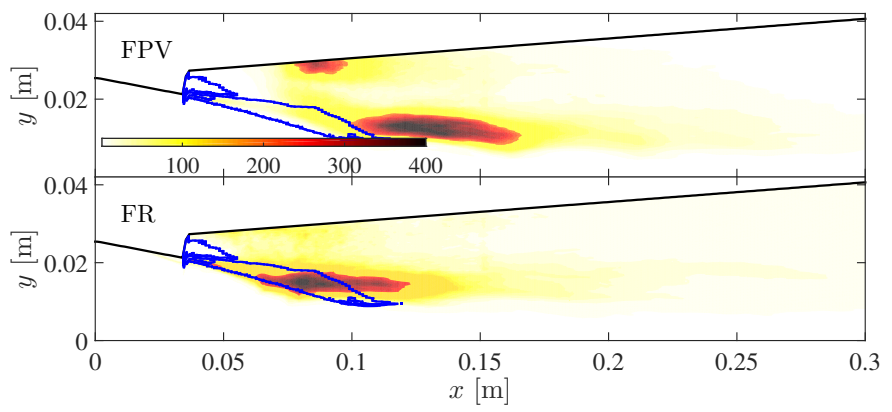

Figure 9. Mean reaction-rate profile of $\widetilde{Y}_{\mathrm{H}_{2} \mathrm{O}}$ of the FPV- (top) and FR-cases (bottom) along the centerplane. The thickened line encloses the region where the mixedness condition, $\widetilde{Z^{\prime \prime 2}}>0.005(\widetilde{Z}(1-\widetilde{Z}))$, is satisfied, delineating the location where TCI is significant in the FPV-results.

The desire for more insights into the characteristics of the UV "A" configuration demands analyses that move beyond comparisons of statistical mean profiles. To do so, the probability density functions (PDF) that underlie the configuration's flameholding recirculation and flame-stabilization zones are evaluated and shown in Figs. 10(a)-10(b), respectively, in terms of the joint PDF of $\widetilde{T}$ with three other variables, namely $\widetilde{Z}, \widetilde{C}$, and $\widetilde{\chi_{Z}}$. Respectively, the physical locations of the two zones are indicated by the windows in Fig. 8 , in the order of increasing $x$. Various laminar flamelet solutions, indicated by lines and symbols, are also provided in Fig. 10 to extract any intrinsic flamelet properties of these joint PDFs. Note that the color scheme of the flamelet solutions follows that of the "S"-shaped curve in Fig. 2, differentiating the upper, middle, and lower branches of the curve. For convention, the upper and lower limits of each branch, in the direction of decreasing temperature along the "S"-shaped curve, are denoted by a solid line or filled square and a dashed line or open square, respectively. The vertical dashed line and circles in the $\widetilde{T}-\widetilde{Z}$ and $\widetilde{T}-\widetilde{C}$ plots, respectively, correspond to the stoichiometric mixture fraction $Z_{\mathrm{st}}=0.0285$. 
Considering the joint PDFs at the flameholding recirculation zone (cf. Fig. 10(a)), the simulation results, regardless of the combustion models, clearly possess strong flamelet-type characteristics that are dominantly relevant to the fuel-rich side of stable upper branch solutions of the "S"-shaped curve. Specifically, the $\widetilde{T}-\widetilde{Z}$ and $\widetilde{T}-\widetilde{C}$ plots consistently reside around the upper limit of the upper branch, exhibiting distributions that agree excellently with the laminar solutions. Scalar-dissipation rate is apparently insignificant at this recirculation region, with no observable probability within the practical range of $0.001<\widetilde{\chi_{Z}}<10001$ /s for the FPV-case and limited distribution within $0.001<\widetilde{\chi_{Z}}<0.011 / \mathrm{s}$ for the FR-case. This combination of low dissipation rate and moderate temperature is indicative of a flame initiation of the autoignitiontype, which is sustained by the constant supply of reaction products, represented by the progress variable, through flow recirculation. For this reason, the recirculation zone has been addressed collectively with its flameholding property throughout this work.

Figure 10(b) refers to the joint PDFs at the flame-stabilization region, which is defined by the approximate spatial range where the mean temperature in both simulations exceed the crossover temperature of $1000 \mathrm{~K}$. The FPV-results, in particular the $\widetilde{T}-\widetilde{Z}$ and $\widetilde{T}-\widetilde{C}$ distributions, remain in good agreement with flamelet solutions of the stable upper branch of the "S"-shaped curve, even though the composition has clearly shifted towards a less fuel-rich regime than seen in Fig. 10(a). In contrast, the $\widetilde{T}-\widetilde{Z}$ plot of the FR-case in Fig. 10(b) only agrees with stable-upper flamelet solutions around the stoichiometric composition and exceeds the maximum flamelet temperature profile (solid red line) for $\widetilde{Z}>0.1$. This increased fuel-rich temperature is possibly related to the aforementioned overprediction in reaction-rate by the FR-results (cf. Figs 8-9), which in turn seems to advocate a lower dissipation rate than the FPV-case in accordance to the $\widetilde{T}-\widetilde{\chi_{Z}}$ plots. Specifically, the scalar-dissipation rate prevails between $[0.01,10] 1 / \mathrm{s}$ and $[0.001,1] 1 / \mathrm{s}$ for the FPV- and FR-cases, respectively. In addition, the $\widetilde{T}-\widetilde{\chi_{Z}}$ distributions suggest that temperature is more correlated to the scalar-dissipation rate in the FPV-case than in the FR-case, as can be deduced from the initial's scattering that is clearly well-fitted by a definite slope of approximately $-270 \mathrm{~K}$. Contrary to their other two joint PDFs, both results appear to be well-represented by the flamelet solutions in the $\widetilde{T}-\widetilde{C}$ plots, implying a general correlation between $\widetilde{T}$ and $\widetilde{C}$ that is indifferent to the combustion model.

\section{Discussions and Future Works}

In Sec. III, we showed through comparisons with measurements that both simulation results are not perfect, exhibiting comparable qualitative and quantitative discrepancies from the experimental results. However, these inaccuracies are expected, given the amount of simplifications that have been included in the boundary conditions and model closures. More importantly, we observed that the essential physical behaviors of the configuration, including the occurrence of an adverse pressure gradient across the combustor, relevance of reactant mixing to reaction-rates, and dependence of flameholding on recirculation of reaction products, are accurately captured by both simulations.

Further analyses that focus on the turbulence combustion models are given in Sec. IV, showing that: (i) the omission of TCI will lead to an overprediction of the reaction-rate that tends to localize near the fuel injection port; and (ii) the combustion models behave similarly, exhibiting a general agreement with the flamelet formulation. Considering the differences in the fundamental assumptions of the two combustion models, which are reflected by the physical discrepancies between the two results shown in Figs. 8-9, the similar flamelet-behavior of the two models is noteworthy, suggesting that the flamelet concept is inherently applicable to the UV "A" configuration, rather than a numerical artifact due to the implementation of flamelet-based models, as in the FPV- case. Therefore, the application of flamelet-type turbulencecombustion models in the current combustor will be valid. However, such application may require the consideration of higher-order effects such as flamelet unsteadiness, as suggested by the significant deviation from the "S"-shaped curve seen in the $\widetilde{T}-\widetilde{\chi_{Z}}$ plots of Fig. 10(b).

In fact, the compatibility of the flamelet concept in the supersonic reacting regime is not novel and has been hypothesized in several previous studies; ${ }^{19-21}$ this work therefore substantiates this postulation with consistent numerical findings. On the other hand, the applicability of the FR-model is likely fortuitous, exploiting the conditions of the UV "A" configuration that apparently tends to localize the effects of TCI. Therefore, the omission of the coupling of turbulence and reaction should be implemented with caution, keeping in mind the potential error that may result from such procedure.

From a cost perspective, the applicability of flamelet-formulation of the UV "A" scramjet combustor renders the FPV-model a suitable closure compared to the FR-approximation, for the reason that the 


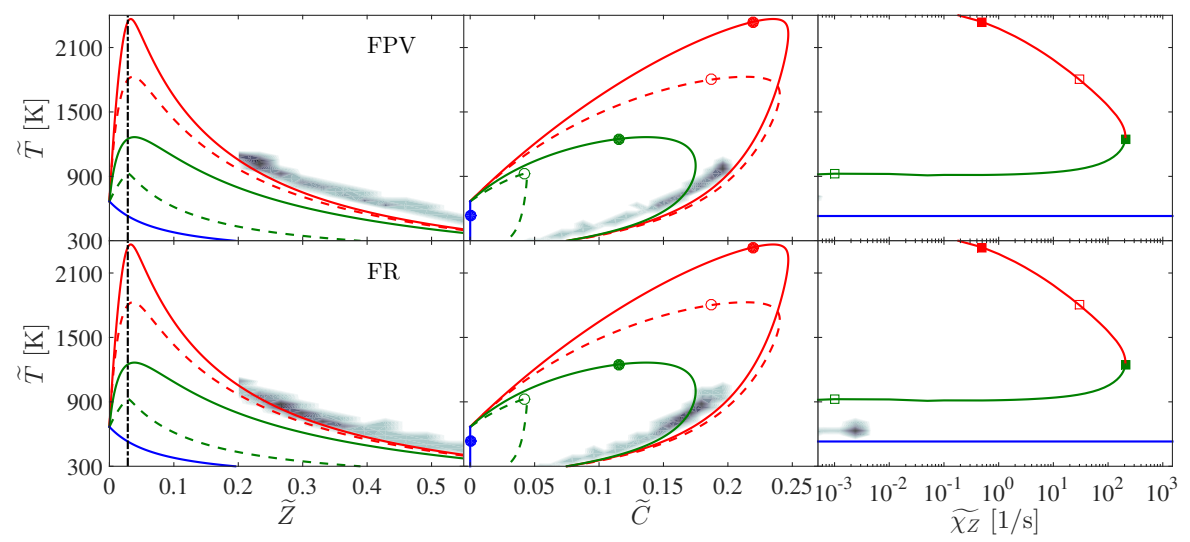

(a)

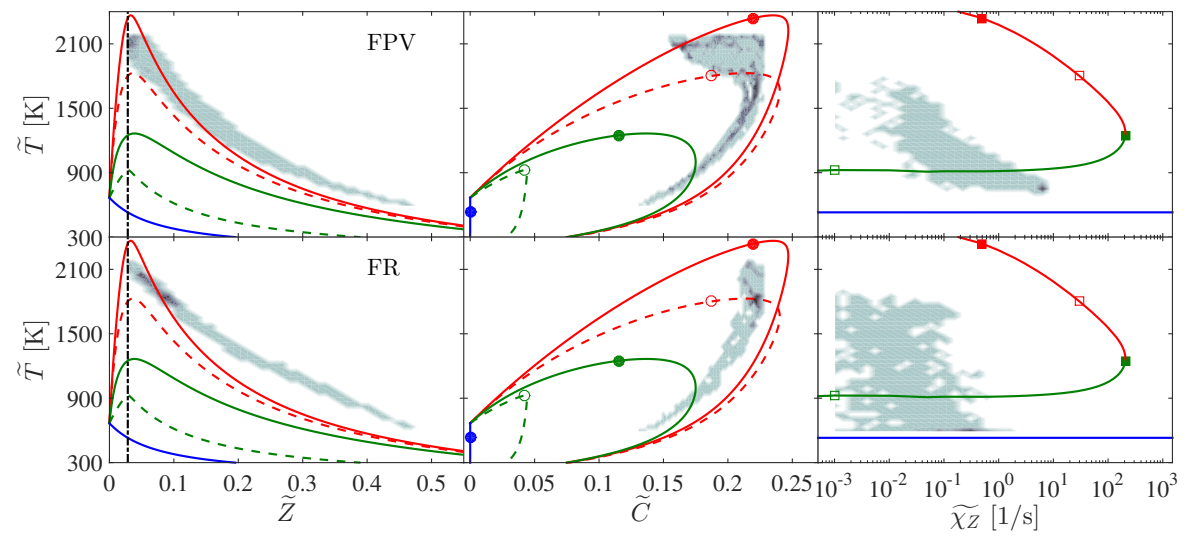

(b)

Figure 10. Joint PDFs of $\widetilde{T}$ with $\widetilde{Z}$ (left), $\widetilde{C}$ (center), and $\widetilde{\chi_{Z}}$ (right) conditioned on the flameholding recirculation (top) and flame-stabilization (bottom) regions. The color scheme of the plots follows the "S"-shaped curve in Fig. 2. The solid and dashed lines correspond to the filled and open squares on the right figure, respectively, while the vertical black line and circles denote the stoichiometric mixture fraction $Z_{\mathrm{st}}=0.0285$.

initial is more efficient (approximately three times in the current work) due to its smaller equation set that significantly reduces system stiffness. Additionally, the FPV-model accounts for TCI through the method of prescribed PDF. ${ }^{22}$

Based on the current findings, the UV "A" configuration is clearly a useful setup for the continued development of flamelet-based combustion models in supersonic reacting regimes. In this regard, aspects of flamelet models that can be extended include the introduction of higher-order flamelet effects ${ }^{23,}{ }^{24}$ formal inclusion of compressibility effects via conditional source-term estimation technique, ${ }^{25}$ and more accurate description of PDF distributions. ${ }^{26,27}$ With regard to improving the simulation predictions, hybrid combustion models of both classes of reaction-transport (e.g. FPV, flame-prologation in intrinsic low-dimensional manifolds ${ }^{28}$ flamelet-generated manifold method $^{29}$ ) and chemistry (i.e. detailed/skeletal reaction kinetics) manifolds can be systematically constructed and optimized using the concept of Pareto-efficiency developed by $\mathrm{Wu}$ et al. ${ }^{30}$ However, prior to such construction of hybrid models, it should be noted that the method's cost and error analysis has to be extended to factor in the effects of TCI, thereby requiring the consideration of more sophisticated TCI closure in the chemistry manifold class than the simple omission of the effect by the FR-approximation; viable ways to account for TCI include the method of approximate reconstruction using moments ${ }^{31}$ and transported PDF. ${ }^{26,32}$

\section{Conclusions}

A numerical study that aims to deepen the understanding of the characteristics of a dual-mode scramjet combustor was conducted. The geometry of the scramjet of interest corresponds to the "A"-configuration of 
the University of Virginia's dual-mode scramjet experiments, which is designed to emulate Mach-5 flight conditions. The simulations were performed in the context of large-eddy simulations. All relevant numerics are kept identical, except for the turbulence combustion model, where the flamelet/progress variable formulation and laminar finite-rate approximation were separately considered.

Through comparison with measurements, both combustion closures were found to perform comparably, generally capturing the essential physical behaviors of the experiments with reasonable accuracies, despite noticeable qualitative and quantitative discrepancies. Due to the differences in the models' underlying assumptions, their similar performance was explored further with model analyses that consist in the comparison of simulation results, quantification of the turbulence/chemistry interaction, and characterization of the configuration's intrinsic flame behaviors. The first two studies essentially showed that the omission of turbulence-reaction coupling will affect the simulation's accuracy by overpredicting the reaction-rate, but this misrepresentation is apparently localized at the upstream fuel injection vicinity, and will eventually be lost as the flow traverse downstream. As a result, a general improvement in both simulations with streamwise distance was observed.

With regard to the flame regime characterization, joint probability density functions of various flow and thermochemical variables were extracted, showing that the flamelet formulation is applicable regardless of the turbulence combustion model. This indifference to the closure model suggests that the flamelet-behavior may be an inherent character of the current configuration. The applicability of flamelet-based combustion models in the supersonic reacting regime is therefore demonstrated. However, the implementation of such models should be cautious against the relevance of higher-order effects, which are conventionally neglected by the models. Such consideration, together with other extensions to the flamelet/progress variable model, is a practical direction for future research to leverage the current findings and scramjet design. In contrast, combustion models of the chemistry manifold class should always consider the coupling of turbulence and reaction for simulation generality, in case the turbulence/chemistry interaction has a more global influence than seen in the current setup.

\section{Acknowledgments}

Financial support through the Air Force Office of Scientific Research is gratefully acknowledged. We would like to thank Brent Rankin for high-performing computational resources that made this work possible.

\section{References}

\footnotetext{
${ }^{1}$ Fureby, C., Tegner, J., Farinaccio, R., Stowe, R., and Alexander, D., "A Computational Study of a Dual-Mode Ramjet Combustor with a Cavity Flameholder," Int. J. Energ. Materials and Chem. Prop., Vol. 11, No. 6, 2012, pp. $487-510$.

${ }^{2}$ Fulton, J. A., Edwards, J. R., Hassan, A. H., McDaniel, J. C., Goyne, C. P., Rockwell, R. D., Cutler, A. D., Johansen, C. T., and Danehy, P. M., "Large-eddy/Reynolds-averaged Navier-Stokes simulations of reactive flow in dual-mode scramjet combustor," J. Prop. Power, Vol. 30, No. 3, 2014, pp. 558-575.

${ }^{3}$ Potturi, A. S. and Edwards, J. R., "Investigation of Subgrid Closure Models for Finite-Rate Scramjet Combustion," 50th AIAA Aerospace Sciences Meeting including the New Horizons Forum and Aerospace Exposition, AIAA 2012-0611, Nashville, TN, 2012.

${ }^{4}$ Saghafian, A., Shunn, L., Philips, D. A., and Ham, F., "Large eddy simulations of the HIFiRE scramjet using a compressible flamelet/progress variable approach," Proc. Combust. Inst., Vol. 35, 2015, pp. 2163-2172.

${ }^{5}$ Fureby, C., "LES for supersonic combustion," 18th AIAA/3AF International Space Planes and Hypersonic Systems and Technologies Conference, AIAA 2012-5979, Tours, France, 2012.

${ }^{6}$ McDaniel, J. C., Chelliah, H., Goyne, C. P., Edwards, J. R., Givi, P., and Cutler, A. D., "US National Center for Hypesonic Combined Cycle Propulsion: An overview," 16th AIAA/DLR/DGLR International Space Planes and Hypersonic Systems and Technologies Conference, AIAA 2009-7280, Bremen, Germany, 2009.

${ }^{7}$ Rockwell Jr., R. D., Goyne, C. P., Rice, B. E., Kouchi, T., McDaniel, J. C., and Edwards, J. R., "Collaborative Experimental and Computational Study of a Dual-Mode Scramjet Combustor," J. Prop. Power, Vol. 30, No. 3, 2014, pp. 530538.

${ }^{8}$ Cutler, A. D., Magnotti, G., Cantu, L., Gallo, E., Rockwell, R., and Goyne, C., "Dual-Pump Coherent Anti-Stokes Raman Spectroscopy Measurements in the Dual-Mode Scramjet," J. Prop. Power, Vol. 30, No. 3, 2014, pp. 539-549.

9 Johansen, C. T., McRae, C. D., Danehy, P. M., Gallo, E. C. A., Cantu, L. M. L., Magnotti, G., Cutler, A. D., Rockwell Jr., R. D., Goyne, C. P., and McDaniel, J. C., "OH PLIF visualization of the UVa supersonic combustion experiment: configuration A," J. Vis., Vol. 17, 2014, pp. 131-141.

$\checkmark{ }^{10}$ Hassan, E., Peterson, M., Tam, C., Eklund, D., and Hagenmaier, M., "RANS-based Multi-Scale Simulations of a Scramjet Combustor," 52nd Aerospace Sciences Meeting, AIAA 2014-0576, National Harbor, MD, 2014.

${ }^{11}$ Chan, W. L. and Ihme, M., "Large-Eddy Simulations of a Dual-Mode Scramjet Combustor: Operating Point "A" of
} 
University of Virginia's Scramjet Experiments," 52nd Aerospace Sciences Meeting, AIAA 2014-1161, National Harbor, MD, 2014.

${ }^{12}$ Pierce, C. D. and Moin, P., "Progress-variable approach for large-eddy simulation of non-premixed turbulent combustion,"

J. Fluid Mech., Vol. 504, 2004, pp. 73-97.

${ }^{13}$ Pečnik, R., Terrapon, V. E., Ham, F., Iaccarino, G., and Pitsch, H., "Reynolds-averaged Navier-Stokes simulations of the HyShot II Scramjet," AIAA J., Vol. 50, 2012, pp. 1717-1732.

${ }^{14}$ Brès, G. A., Ham, F. E., Nichols, J. W., and Lele, S. K., "Nozzle wall modeling in unstructured large eddy simulations for hot supersonic jet predictions," 19th AIAA/CEAS Aeroacoustics Conference, AIAA 2013-2142, Berlin, Germany, 2013.

$\checkmark{ }^{15}$ Burke, M. P., Chaos, M., Ju, Y., Dryer, F. L., and Klippenstein, S. J., "Comprehensive H2/O2 Kinetic Model for High-Pressure Combustion," Int. J. Chem. Kinet., Vol. 44, No. 7, 2011, pp. 444-464.

16 Vreman, A. W., "An eddy-viscosity subgrid-scale model for turbulent shear flow: Algebraic theory and applications," Phys. Fluids, Vol. 16, No. 10, 2004, pp. 3670-3681.

${ }^{17}$ Hewson, J. C. and Kerstein, A. R., "Local extinction and reignition in nonpremixed turbulent CO/ $\mathrm{H}_{2} / \mathrm{N}_{2}$ jet flames," Comb. Theory Modelling, Vol. 174, 2002, pp. 35-66.

${ }^{18}$ Pope, S. B., "Ten questions concerning the large-eddy simulation of turbulent flows," New J. Phys., Vol. 6, No. 35, 2004.

${ }^{19}$ Ladeinde, F., "A critical review of scramjet combustion simulation (Invited)," 47th AIAA/ASM including The New Horizons Forum and Aerospace Exposition, AIAA 2009-127, Orlando, FL, 2009.

${ }^{20}$ Ingenito, A. and Bruno, C., "Physics and regimes of supersonic combustion," AIAA J., Vol. 48, No. 3, 2010 , pp. 515-525.

${ }^{21}$ Fulton, J. A., Edwards, J. R., Cutler, A., McDaniel, J., and Goyne, C., "Turbulence/Chemistry Interactions in a RampStabilized Supersonic Hydrogen-Air Diffusion Flame," 52nd Aerospace Sciences Meeting, AIAA 2014-0627, National Harbor, MD, 2014.

${ }^{22}$ Peters, N., Turbulent Combustion, Cambridge University Press, 2000.

${ }^{23} \mathrm{Ihme}, \mathrm{M}$. and See, Y. C., "Prediction of autoignition in a lifted methane/air flame using an unsteady flamelet/progress variable model," Combust. Flame, Vol. 157, 2010, pp. 1850-1862.

-24Scholtissek, A., Chan, W. L., Xu, H., Hunger, F., Kolla, H., Chen, J. H., Ihme, M., and Hasse, C., "A multi-scale asymptotic scaling and regime analysis of flamelet equations including tangential diffusion effects for laminar and turbulent flames," Combust. Flame, Vol. 162, 2015, pp. 1507-1529.

${ }^{25}$ Labahn, J. W., Dovizio, D., and Devaud, C. B., "Numerical simulation of the Delft-Jet-in-Hot-Coflow (DJHC) flame using Conditional Source-term Estimation," Proc. Combust. Inst., Vol. 35, 2015, pp. 3547-3555.

${ }^{26}$ Koo, H., Donde, P., and Raman, V., "A quadrature-based LES/transported probability density function approach for modeling supersonic combustion," Proc. Combust. Inst., Vol. 33, 2011, pp. 2203-2210.

$\checkmark{ }^{27}$ Coclite, A., Pascazio, G., De Palma, P., Cutrone, L., and Ihme, M., "An SMLD Joint PDF Model for Turbulent NonPremixed Combustion using the Flamelet Progress-Variable Approach," Flow, Turb. Combust., Vol. 95, 2015, pp. 97-119.

${ }^{28}$ Gicquel, O., Darabiha, N., and Thévenin, D., "Laminar premixed hydrogen/air counterflow flame simulations using flame prolongation of ILDM with differential diffusion," Proc. Combust. Inst., Vol. 28, 2000, pp. 1901-1908.

${ }^{29}$ Nguyen, P.-D., Vervisch, L., Subramanian, V., and Domingo, P., "Multidimensional flamelet-generated manifolds for partially premixed combustion," Combust. Flame, Vol. 157, 2010, pp. 43-61.

${ }^{30} \mathrm{Wu}, \mathrm{H}$., See, Y. C., Wang, Q., and Ihme, M., "A Pareto-efficient combustion framework with submodel assignment for predicting complex flame configurations," Combust. Flame, Vol. In press, 2015.

31 Mellado, J. P., Sarkar, S., and Pantano, C., "Reconstruction subgrid models for nonpremixed combustion," Phys. Fluids, Vol. 15, No. 11, 2003, pp. 3280-3307.

${ }^{32}$ Colucci, P. J., Jaberi, F. A., Givi, P., and Pope, S. B., "Filtered density function for large eddy simulation of turbulent reacting flows," Phys. Fluids, Vol. 10, No. 2, 1998, pp. 499-515. 
This article has been cited by:

1. Ez Hassan, Edward A. Luke, D. Keith Walters, David M. Peterson, Dean Eklund, Mark Hagenmaier. 2017. Computations of a Hydrogen-Fueled Scramjet Combustor on Locally Refined Meshes. Flow, Turbulence and Combustion 99:2, 437-459. [Crossref]

2. Esteban D. Gonzalez-Juez, Alan R. Kerstein, R. Ranjan, S. Menon. 2017. Advances and challenges in modeling high-speed turbulent combustion in propulsion systems. Progress in Energy and Combustion Science 60, 26-67. [Crossref] 